\title{
The UDP-glucose ceramide glycosyltransferase (UGCG) and the link to multidrug resistance protein 1 (MDR1)
}

\author{
Marthe-Susanna Wegner ${ }^{1 *} \mathbb{D}$, Lisa Gruber ${ }^{1}$, Peter Mattjus ${ }^{2}$, Gerd Geisslinger ${ }^{1}$ and Sabine Grösch ${ }^{1}$
}

\begin{abstract}
The UDP-glucose ceramide glycosyltransferase (UGCG) is a key enzyme in the sphingolipid metabolism by generating glucosylceramide (GlcCer), the precursor for all glycosphingolipids (GSL), which are essential for proper cell function. Interestingly, the UGCG is also overexpressed in several cancer types and correlates with multidrug resistance protein 1 (MDR1) gene expression. This membrane protein is responsible for efflux of toxic substances and protects cancer cells from cell damage through chemotherapeutic agents. Studies showed a connection between UGCG and MDR1 overexpression and multidrug resistance development, but the precise underlying mechanisms are unknown. Here, we give an overview about the UGCG and its connection to MDR1 in multidrug resistant cells. Furthermore, we focus on UGCG transcriptional regulation, the impact of UGCG on cellular signaling pathways and the effect of UGCG and MDR1 on the lipid composition of membranes and how this could influence multidrug resistance development. To our knowledge, this is the first review presenting an overview about UGCG with focus on the relationship to MDR1 in the process of multidrug resistance development.
\end{abstract}

Keywords: UDP-glucose ceramide glycosyltransferase, UGCG, Glycosphingolipids, MDR1, P-gp, Multidrug resistance, Cancer

\section{Background}

Over the last two decades, researchers were able to link the sphingolipid metabolism and the development of multidrug resistance in several cancer types, but the precise mechanisms are unknown. There is evidence that the UDP-glucose ceramide glycosyltransferase (UGCG), which is located in the Golgi apparatus (Fig. 4) is involved in the process of multidrug resistance development. The UGCG was first cloned by Ichikawa et al. in the year 1996 [1] and is currently the only enzyme responsible for the de novo production of glucosylceramide (GlcCer). GlcCer is the precursor for all glycosphingolipids (GSLs) such as globo-series and ganglio-series GSLs. Since more than 400 species of GSLs are identified, the GSL metabolic pathway is here described only briefly (reviewed in [2]). Ceramides, generated in the endoplasmic reticulum by ceramide

\footnotetext{
* Correspondence: wegner@med.uni-frankfurt.de

1 pharmazentrum frankfurt/ ZAFES, Institute of Clinical Pharmacology, Johann Wolfgang Goethe-University, House 74, Theodor Stern-Kai 7, 60590 Frankfurt am Main, Germany

Full list of author information is available at the end of the article
}

synthases (CerS), are transported to the Golgi apparatus, where the UGCG transfers UDP-glucose to ceramide resulting in GlcCer. Subsequently, the produced GlcCer is flipped from the cytosol to the Golgi apparatus lumen for lactosylceramide (LacCer) production by galactosylation of GlcCer. LacCer serves as a substrate for a variety of GSLs producing enzymes (reviewed in [2]). From the Golgi apparatus GSLs are transported to the plasma membrane, where they are integrated (Fig. 4). GSLs depletion in the plasma membrane is executed by endocytotic membrane flow of GSLs into the lysosomes, where glycohydrolase activity results in GSLs degradation and subsequently ceramide production [3]. GSL are essential for embryonic development and differentiation of several tissue types [4] and are involved in lipid storage disorders called sphingolipidoses, which are caused by deficiency of lysosomal enzymes. One of the main sphingolipidoses is the Gaucher disease, which is characterized by deficiency of glucocerebrosidase leading to GlcCer accumulation in cells and organs. Due to different phenotypes the clinical presentation of Gaucher disease patients ranges from cytopenia to neurological 
impairment (reviewed in [5]). In patients with Fabry disease an GSL accumulation (in general glycolipids) occurs also, but in this case due to mutations in the GLA gene encoding $\alpha$-galactosidase A (reviewed in [6]). The Sandhoff and Tay Sachs disease are characterized by accumulation of the GSL GM2 leading to neurodegeneration and early death (reviewed in [7]). Although, the phenotype of the Niemann-Pick disease is based on deficient sphingomyelinase activity, GlcCer concentration is found to be the second highest lipid concentration [8]. The different types of sphingolipidoses show how important it is to understand the sphingolipid metabolism and how complex it is. To date, only few reviews dealing with the UGCG are available [9-11], therefore we will discuss the impact of the UGCG and its knockout on cell proliferation and cell metabolism shortly.

A knockout of the UGCG in mice leads to embryonic lethality, which occurs at the phase of gastrulation. However, Yamashita et al. showed a not discriminable proliferation rate between UGCG deleted embryonic stem cells and wildtype cells in vitro, although GSL synthesis was absent in the UGCG disrupted embryonic stem cells [4]. Instead, nude mice studies showed that silencing the UGCG gene in adriamycin-resistant MCF-7 cells, which overexpress the UGCG, inhibits tumor xenograft growth in vivo [12]. This result is in line with the studies of the Sabatini Lab (Whitehead Institute, Cambridge, Massachusetts) showing that the UGCG is an essential protein for optimal proliferation of several cancer cell lines [13]. The working group developed a Clustered Regularly Interspaced Short Palindromic Repeats (CRISPR) score (CS), which mirrors the loss of fitness evoked by knockout of the respective gene. The CS is represented „as the average log2 fold-change in the abundance of all sgRNAs targeting a given gene" and indicates whether the gene knockout leads to an increased or decreased cancer cell number. For the UGCG the following CS were determined: KBM7 (-0,243), K562 $(0,163)$, Jiyoye $(-0,08)$ and Raji $(0,364)$ cells [13]. These values show that the UGCG is essential for cell proliferation, but how severe the impact on the cell metabolism is seems to be cell-type specific. In summary, contradictory results were obtained regarding the essentiality of the UGCG gene for cell proliferation. Another explanation for the contradictory results could be the existence of a second form of this protein, which balances the loss of the other protein. But our high-performance thinlayer chromatography (HPTLC) studies show that there is no compensation of GlcCer loss in UGCG deficient MCF-7 cells (lane 5) (Fig. 1). Therefore, UGCG may be a unique protein in cells, solely responsible for the first glycosylation step of ceramides leading to complex GSLs. Accordingly, knockdown of the UGCG impacts all other glycosylated sphingolipids such as LacCer, the

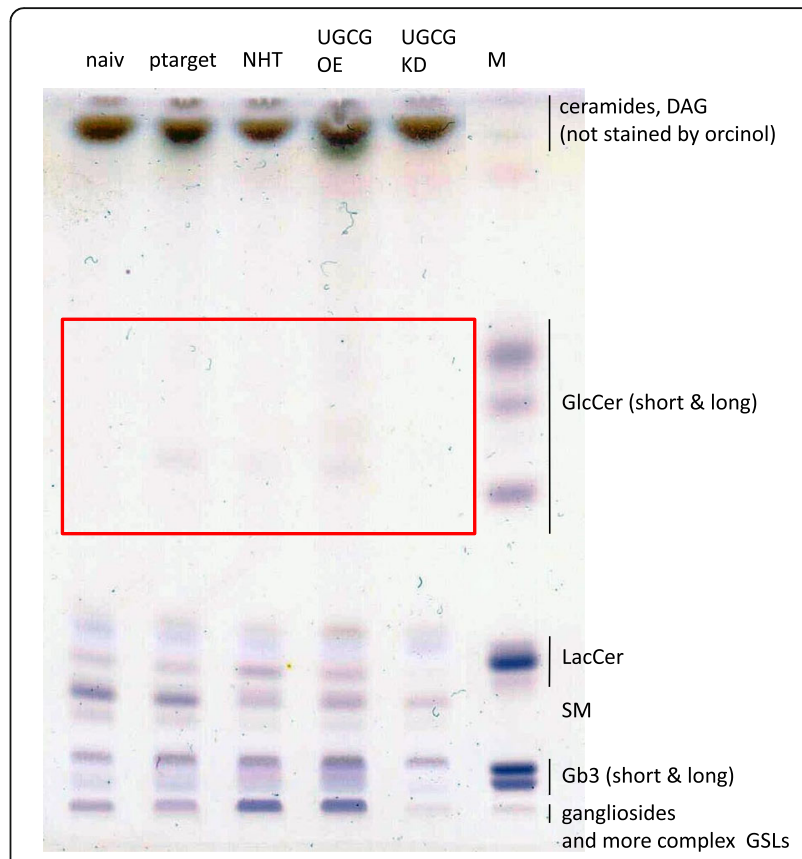

Fig. 1 High-performance thin-layer chromatography (HPTLC) analysis of diverse sphingolipid species in MCF-7 cells. Representative HPTLC plate showing the separation of ceramides, DAG and non-complex GSLs like GlcCer, LacCer and complex GLSs like Gb3 and gangliosides. MCF-7/naiv: untransfected, MCF-7/pTarget: empty vector-transfected, MCF-7/NHT: CRISPR-Cas non-human target control, MCF-7/UGCG OE: UGCG expression plasmid-transfected, MCF-7/KD: CRISPR-Cas UGCG Knockdown. $\mathrm{M}=$ Marker

globo-series sphingolipid globotriaosylceramide (Gb3), gangliosides and complex GSLs, which are lower in its concentration in MCF-7 UGCG knockout cells (Fig. 1).

Disruption of the UGCG in mice epidermis, causes alterations in the skin lipid composition leading to the loss of the barrier function and to death due to dehydration [14]. GlcCer is needed for proper lamellar body formation and regular metabolism in the stratum corneum, which is essential for maintaining water permeability function. Amen et al. showed that an inducible UGCG knockout in mice keratinocytes leads to a loss of protein-bound ceramides resulting in the same fate, that occurs to the constitutive knockout mice [15].

Beside its essential functions especially in the maintenance of the epidermal water barrier function, UGCG is overexpressed in several cancer types for example in metastatic breast cancer tissue leading to poor patient prognosis [16] (Fig. 4). Gouazé et al. showed that this is not a unique phenomenon for breast cancer cells, because several other cancer cells e.g. human leukemia and colon cancer cells, also exhibit a high UGCG gene expression [17]. This overexpression correlates with increased multidrug resistance protein 1 (MDR1) gene expression, which encodes the protein P-glycoprotein 1 (P-gp) (also ATP-binding cassette sub-family B member 
1, ABCB1), leading to multidrug resistance for example against vinblastin (human cervix carcinoma KB-3-1 cells; Hela derivatives), vincristine (human leukemia (HL)-60 cells) and adriamycin (human colon cancer cells SW620). UGCG blockage resensitizes multidrug resistant breast cancer cell to anticancer drugs via downregulation of MDR1 [18].

The resistance of tumors to chemotherapeutic agents occurs via highly complex mechanisms and is a serious challenge in cancer therapy. The P-gp protein expressed at the plasma and Golgi membrane plays an important role in multidrug resistance by transporting toxic substances out of the cell (reviewed in [19]) (Fig. 4). This efflux pump, which is also expressed in normal tissue for protection from toxic substances, transports hydrophobic substances like nonylphenol ethoxylate (component of human urine) (reviewed in [20]). In addition, the P-gp protein transports hydrophobic substances like anthracyclines, taxanes and kinase inhibitors. P-gp is regulated by a variety of mechanisms on transcriptional and posttranscriptional level like extracellular-signal regulated kinase (ERK) signaling, Skp1-Cullin1-FBX015 $\left(\mathrm{SCF}^{\mathrm{FBX} 15}\right)$ complex and glycogen synthase kinase 3 (GSK-3), which transcriptionally inhibits MDR1 through wingless $(\mathrm{Wnt}) / \beta$-catenin signaling (reviewed in [19]), but also via ubiquitin-proteasomal degradation by action of the ubiquitin-activating enzyme E2 R1 (UBE2R1). For more detailed information of P-gp please see the following reviews: $[21,22]$.

The UGCG provides a major route for ceramide clearance in the case of an increased activity of CerS6, which generates the pro-apoptotic $\mathrm{C}_{16: 0}$-Cer, accordingly, ceramide induced apoptosis is not induced. It is likely, that in the case of extrinsic and intrinsic apoptosis pathway the UGCG is not capable to clear the ceramide due to its localization. Through extracellular signals like oxidative stress or Tumor necrosis factor $\alpha$ (TNF $\alpha$ ) cellular ceramide concentration increases and induces apoptosis. Ceramides can either be generated by the activation of the sphingolipid de novo synthesis, which occurs in the endoplasmic reticulum or by induction of the salvage pathway e. g. by hydrolysis of sphingomyelin. Initiation of the extrinsic apoptosis signaling pathway, occurs by ligand binding to the Tumor necrosis factor receptor 1 (TNFR1, death receptor). Subsequently, receptor trimerization and activation of the protein Factor associated with activation of neutral sphingomyelinase (SMase) (FAN) that binds to the intracellular neutral SMase domain (NSD), thereby activating the neutral SMase, occurs. The hydrolysis of sphingomyelin in the plasma membrane leads to $\mathrm{C}_{16: 0^{-}}$-ceramide release, which activates caspase-dependent apoptosis (reviewed in [23]). The UGCG seems not to be capable to logistically reach the ceramide for clearing it into less cytotoxic molecules. Also in the case of the intrinsic apoptotic pathway, which recently was shown to be mediated by a mitochondria-specific ceramide pool (reviewed in [24]), the residence of the UGCG in the Golgi apparatus seems to prohibit ceramide clearance.

\section{Materials and methods Cell culture}

The human breast adenocarcinoma cell line MCF-7 were purchased from the Health Protection Agency (catalogue number: 86,012,803, European Collection of Cell Cultures, ECACC, Salisbury, UK) and was cultured in Dulbecco's Modified Eagle Medium (DMEM) containing high glucose, no phenol-red and no HEPES. 1\% GlutaMAX, $1 \%$ sodium pyruvat and 5\% charcoaled fetal bovine serum (FBS) (Sigma-Aldrich, Deisenhofen, Germany) was added. Cells were incubated at $37{ }^{\circ} \mathrm{C}$ in an atmosphere containing $5 \% \mathrm{CO}_{2}$. For selection of stably transfected cells, G418 (Thermo Fisher Scientific, Waltham, Massachusetts, USA) was added.

\section{Stable transfection of UDP-glucose ceramide glucosyl-} transferase (UGCG) expression plasmid in MCF-7 cells UDP-glucose ceramide glucosyltransferase (UGCG) expression plasmid (pCMV6-ENTRY vector) was purchased from OriGene Technologies Inc. (Rockville, USA). The pTarget empty vector was purchased from Promega GmbH (Mannheim, Germany). Stable transfection was performed with Lipofectamine 2000 Reagent (Invitrogen by Life Technologies) according to the manufacturer's protocol. MCF-7 cells were transfected with $2 \mu \mathrm{g}$ of the distinct plasmid and selected over 5 weeks with different G418 concentrations. Under a G418 concentration of $400 \mu \mathrm{g} / \mathrm{ml}$ all untransfected cells died, subsequently, this concentration was considered to be sufficient for culturing the stable transfected cells.

\section{Sphingolipid concentration determination by high- performance thin-layer chromatography (HPTLC)}

All chemical reagents were of analytical grade or higher. Lipid standards where from Avanti Polar Lipids (Alabaster, USA) or Matreya LLC (Pleasant Gap, USA). Organic solvents were from Rathburn Chemicals Ltd. (Walkerburn Scotland). The high performance thin-layer chromatography (HPTLC) silica plates were from Whatman, UK. $9 \times 10^{6}$ MCF-7 cells were scraped in $1 \times$ PBS, centrifuged and frozen in liquid nitrogen. Cells were dried under the hood for three days and stored in a freezer $\left(-80{ }^{\circ} \mathrm{C}\right)$ until analyzed. Each dried sample was solubilized in ice cold MQ-water $(1 \mathrm{ml})$ by bath sonication (ice-water bath) for $5 \mathrm{~min}$. The solubilized samples were transferred to clean glass test tubes (with screw-caps). 
Total lipids were extracted by a modified Blight and Dyer protocol: $1 \mathrm{ml}$ of MQ-water was added to each sample (2 $\mathrm{ml}$ of total water volume), $3 \mathrm{ml}$ of chloroform:isopropanol (2:1, vol:vol) was added to each sample, samples were vortexed thoroughly for $10 \mathrm{~s}$, and rotated end-over-end for $20 \mathrm{~min}$ at RT. The samples were centrifuged at $4.000 \mathrm{rpm}$ for $20 \mathrm{~min}$ at RT to separate the phases. The organic phase (bottom) was carefully extracted with a glass Pasteur pipette and placed in a clean glass tube, without disturbing the protein precipitate at the interphase. The chloroform:isopropanol (2:1, vol:vol) extractions were repeated once, as described above, and combined with the previous extracts. $3 \mathrm{ml}$ of hexane was then added to each sample, samples were vortexed, rotated and centrifuged as described above. The hexane phases (top) were extracted and combined with the previous chloroform:isopropanol extracts. The organic solvent containing the extracted lipids was dried under a stream of nitrogen. The dry samples were stored at $20{ }^{\circ} \mathrm{C}$. Each of the dried lipid sample was resolubilized in an appropriate volume of chloroform:isopropanol (2:1, vol:vol). The lipid samples were normalized according to their corresponding protein concentrations and applied on an HPTLC plate using an autosampler. The HPTLC plate was developed using the solvent system chloroform:methanol:acetone:acetic acid:water (10:2:4:2:1). The sugar residues of the glycolipids were visualized by spraying the plate with an orcinol solution $(0.3 \%$ orcinol in $20 \%$ sulphuric acid) and by heating $\left(<5 \mathrm{~min}, 120{ }^{\circ} \mathrm{C}\right)$. Lipids were identified with the help of commercial standards that were run in parallel with the samples on the HPTLC plate.

The precipitated protein was isolated as follows: $1 \mathrm{ml}$ of chloroform and $1.5 \mathrm{ml}$ of methanol was added to the remaining aqueous phases of the samples. Samples were briefly vortexed and centrifuged at $4.000 \mathrm{rpm}$ for $20 \mathrm{~min}$. The aqueous upper phase was carefully removed with a glass Pasteur pipette and discarded, leaving the interphase (with the precipitated proteins) untouched. $2 \mathrm{ml}$ of methanol was added to each of the samples. Samples were again briefly vortexed and centrifuged at $4.000 \mathrm{rpm}$ for $20 \mathrm{~min}$, to pellet the precipitated proteins. The chloroform:methanol solution was carefully removed and discarded with a glass Pasteur pipette, without disturbing the pelleted proteins. The pellets were carefully dried to completion, under a stream of nitrogen. Each protein sample was re-solubilized in $500 \mu \mathrm{l}$ of an $8 \mathrm{M}$ urea solution (8 $\mathrm{M}$ urea in PBS, $\mathrm{pH} 6.8,0.5 \%$ SDS) by vigorous vortexing at RT. The protein samples were stored at $-20{ }^{\circ} \mathrm{C}$. The concentrations of the solubilized proteins were determined by the method of Lowry.

\section{Retrospective analysis UGCG and MDR1 Upstream effectors}

UGCG promoter methylation Recently, Liu et al. [25] showed that the $\mathrm{CpG}$ island methylation at the UGCG promoter (Fig. 2) correlates inversely with its gene expression and multidrug resistance development in invasive ductal breast cancer tissue. UGCG CpG island methylation was negatively associated with estrogen receptor (ER) positive status (ER $\alpha$ expressing) and positively associated with HER-2 positive status [25]. In addition, in vitro experiments showed that 5-Aza-2 deoxycytidine (decitabine; DNA methylation inhibitor) could reverse the DNA methylation status of the UGCG promoter, which restores UGCG gene expression and correlates with multidrug resistance development. The study also showed that the methylation status of the UGCG promoter is related to the expression of $D N A$ (cytosine-5)-methyltransferase 3a (DNMT3a) (Fig. 2), which catalyzes the transfer of methyl groups to $\mathrm{CpG}$ structures in the DNA [25]. However, previous data from the same group showed a correlation of UGCG expression with ER positive and HER-2 positive status in breast cancer cells [26]. Since the DNMT3a encoding gene is frequently mutated in several cancer types [27], more studies are needed to shed light on the DNMT3aUGCG-P-gp axes. Studies from Raghavan et al. show that beside UGCG, also other enzymes involved in the lipid metabolism are methylation-dependently regulated and thus leading to altered sensitivity against several drugs [28]. The cell membrane composition of doxorubicin resistant MCF-7 cells differs from membrane composition of sensitive MCF-7 cells (Fig. 4). E. g. cell membranes of resistant cells exhibit an increased sphingomyelin concentration [28]. By decitabine treatment the changes in resistant MCF-7 cell membranes could partially be reversed. The question remains whether the altered membrane composition leads to multidrug resistance induction by altering gene expression or the overexpression of the UGCG induces altered membrane composition and thus leading to multidrug resistance.

P-gp and others on UGCG promoter In 2012, studies showed that doxorubicin treatment leads to an increase of UGCG mRNA and protein concentration in ER positive MCF-7 cells, but only to a slight increase in ER negative MDA-MB-231 cells [29]. This is accompanied by development of resistance to doxorubicin treatment. When the transcription factor Sp1, which has a binding site at the UGCG promoter, is inhibited by Sp1 decoy ODNs transfection, doxorubicin does not lead to an increased UGCG mRNA and protein expression in MCF-7 


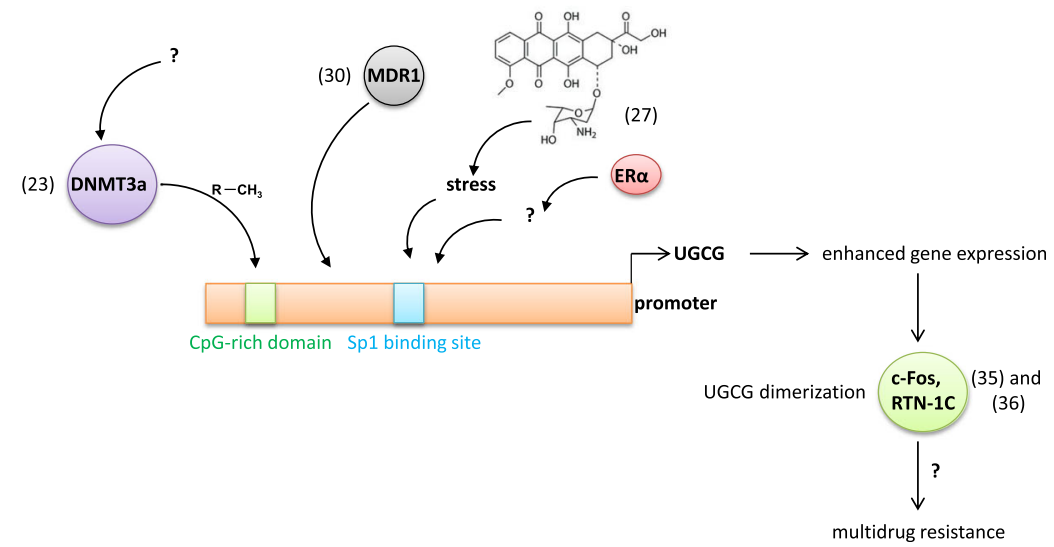

Fig. 2 Upstream effectors - Schematic display of UGCG promoter activity regulating processes in the context of multidrug resistance. DNMT3a = DNA (cytosine-5)-methyltransferase 3a, $\mathrm{CH}_{3}=$ methyl group, MDR1 = multidrug resistance protein 1, ERa=estrogen receptor $a$, UGCG = UDP-glucose ceramide glycosyltransferase, RTN-1C = Reticulon-1C

cells (Fig. 2). The doxorubicin resistance is reversed and apoptotic events occur. Additionally, blocking the ER subtype $\alpha(E R \alpha)$ also prevents UGCG upregulation after doxorubicin treatment (Fig. 2) [29]. In ER positive MCF7 cells blocking of ER $\alpha$ has a severe impact on the cell metabolism, since ER $\alpha$ promotes cell proliferation by direct transcriptional upregulation of several proliferation promoting genes like $M y c, B$-cell lymphoma 2 (BCL2) and E2F transcription factor 1 (E2F1) and indirectly like FOS (reviewed in [30,31]). This led us to suggest, that the decrease of UGCG mRNA after ER $\alpha$ inhibition and induction of apoptosis after doxorubicin treatment may be based on an entire block of the cell metabolism. In addition, although in the study from Zhang et al. Sp1 decoy ODNs were used, which contain the sequence of the UGCG putative Sp1/GC-rich binding site, it cannot be excluded that another Sp1regulated gene is blocked in its expression, which could be responsible for the UGCG promoter activity increase. If this is the case, the altered UGCG promoter activity is only a downstream effect and not directly mediated by $\mathrm{Sp} 1$. The same might be true for the ER $\alpha$ effect. It is also possible that additional transcription factors to $\mathrm{Sp} 1$ are involved in UGCG promoter activity regulation. Therefore, it would be helpful to perform reporter gene studies with deleted transcription factor binding sites of the UGCG promoter to verify this result. In addition, DNA-protein interactions of the UGCG promotor sequence and e.g. Sp1 should be monitored by chromatin immunoprecipitation (ChIP) assays or by electrophoretic mobility shift assay (EMSA) to proof the interaction. Furthermore, it has to be clarified how the doxorubicin effect on the UGCG promoter is exactly mediated since this chemotherapeutic agent leads to cell stress by induction of reactive oxygen species (ROS) or p53 accumulation and subsequently induces apoptotic signaling pathways. However, since doxorubicin is a known inducer of P-gp it has to be clearified whether or not this induction is mediated via a doxorubicin-dependent activation of UGCG gene transcription resulting in an UGCG-regulated MDR1 gene expression (Fig. 4). Whether or not this mechanism is possible for other Pgp inducer or inhibitors such as verapamil or ciclosporin should be investigated in the future.

Most studies show an UGCG-dependent regulation of $M D R 1$ in the context of multidrug resistance induction (Fig. 3). However, studies by Zhang et al. revealed that this dependency occurs possibly vice versa, meaning that P-gp can directly influence UGCG expression in breast cancer cells [32] (Fig. 2). Zhang et al. showed that treatment of adriamycin resistant MCF-7 cells, which exhibit an increased MDR1 and UGCG expression as compared to control cells, with siRNA against MDR1 results in decreased MDR1 and UGCG mRNA expression and protein concentration indicating that MDR1 regulates UGCG expression. How exactly this UGCG upregulation is accomplished by MDR1 has not been investigated.

\section{Downstream effectors}

Induction of apoptosis and potential heterodimerization of UGCG

Most studies published in the last two decades, which investigated the involvement of the UGCG and MDR1 in multidrug resistance, have focused on the association 
Downstream effectors

a

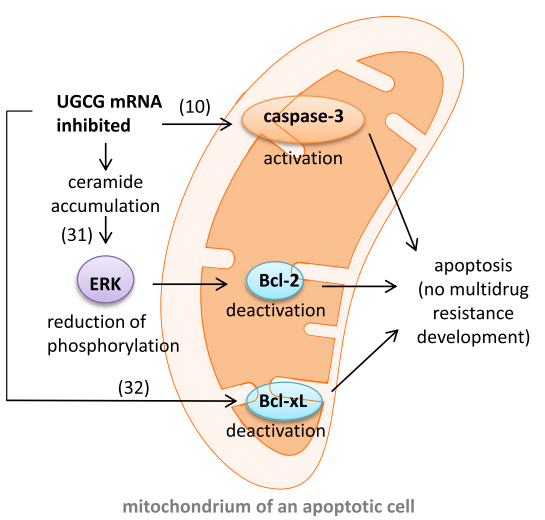

b

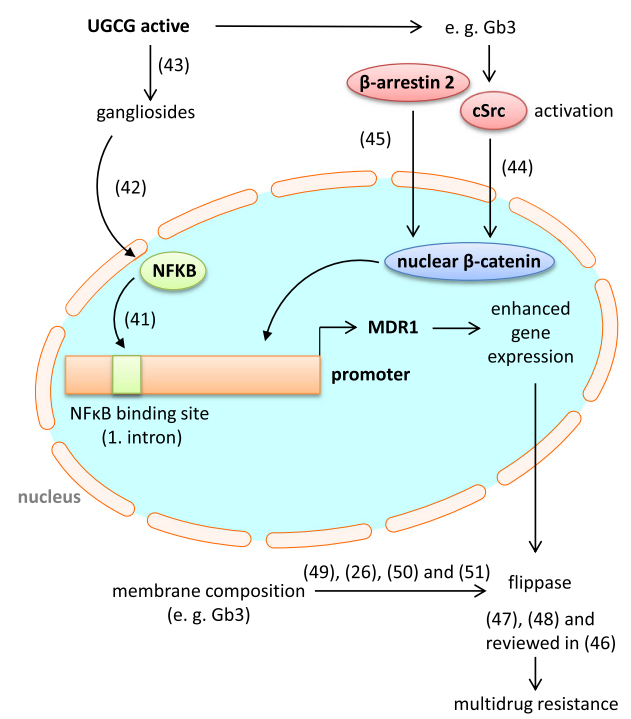

Fig. 3 Downstream effectors - Schematic display of UGCG regulated processes in the context of multidrug resistance. a UGCG mRNA inhibition leads to induction of apoptotic processes and prevents multidrug resistance development. b UGCG activity derived GSLs such as gangliosides regulate MDR1 promoter activity. UGCG $=U D P$-glucose ceramide glycosyltransferase, $\mathrm{ERK}=$ extracellular-signal regulated $\mathrm{kinase}, \mathrm{BCl}-2=B$-cell lymphoma 2, Bcl-xL = B-cell lymphoma-extra large, NFKB = nuclear factor (NF) $\mathrm{kB}, \mathrm{cSrc}=$ tyrosine kinase, Gb3 = Globotriasoylceramide, $\mathrm{MDR} 1=$ multidrug resistance protein 1

of UGCG and its downstream effectors. Progress was made regarding the elucidation of processes following UGCG inhibition, especially processes leading to an induction of apoptotic signaling pathways. In 2010 already, Sun et al. showed that inhibition of the UGCG leads to high ceramide levels in breast cancer cells and results in caspase- 3 activation and subsequently cell death (Figs. 3a and 4). This study also demonstrated that in a mice xenograft model treatment with shRNA against the UGCG leads to decreased tumor growth and that beside the UGCG also the MDR1 gene expression and subsequently P-gp protein is inhibited in vivo [12]. Years later Wang et al. showed that UGCG knockdown decreases the mRNA and protein levels of Bcl-2 (anti-apoptotic) via reduction of ERK phosphorylation, which was induced by ceramide accumulation in K562/A02 cells [33] (Fig. 3a). Beside Bcl-2, also B-cell lymphoma-extra large (Bcl-xL), which has an anti-apoptotic effect by preventing e. g. cytochrome $\mathrm{c}$ release from mitochondria, is decreased in its expression when the UGCG is inhibited [34] (Fig. 3a). However, Liu et al. published that MCF-7 cells, which overexpress the UGCG are resistant against adriamycin and ceramide toxicity, but P-gp was not detectable [35]. In addition, the phosphorylation status of the anti-apoptotic $\mathrm{Bcl}-2$ protein was not altered indicating that the multidrug resistance in these cells is independent from both, MDR1 and $\mathrm{Bcl}-2$. It is possible that the sphingolipid signaling pathway is the main factor in this process, because the UGCG overexpressing cells showed only a slight increase in GlcCer amount (see also Fig. 1). This may be due to a low substrate supply or low UGCG activity, a fast processing of GlcCer to complex GSLs or degradation of GlcCer. Furthermore, the UGCG can interact with other unknown proteins and induces thereby the drug resistance. It was already shown that UGCG protein from rat is organized as a heterodimer or heterooligomer [36]. Approved binding partners for the UGCG are c-Fos [37] and the neuroendocrine-specific protein Reticulon-1C [38], whereby the UGCG activity does not depend on complex formation with these proteins (Figs. 2 and 4). Future studies should investigate the influence of these complexing partners in the process of multidrug resistance. The idea of UGCG protein interaction with another protein could be supported by a study showing that curcumin inhibits UGCG activity in drug-resistant cells only. This means that the multidrug resistant cells express proteins, which mediate the curcumin-induced UGCG inhibition [39]. In Hela cells only the combination of mifepristone (progesterone receptor antagonist) and mitomycin c (DNA synthesis block) inhibited UGCG [40], meaning that after mitomycin c treatment one or more specific genes are not transcribed, which are essential for induction of multidrug resistance. It is possible that also after curcumin 




Fig. 4 Summary of the molecular mechanisms regarding the connection between UGCG and MDR1 in the process of multidrug resistance development. In sensitive cells (green) cytotoxic agents lead to apoptosis by, amongst other mechanisms, UGCG gene expression inhibition and following ceramide accumulation. During the process of multidrug resistance development (red) cytotoxic agents may lead to an increased gene expression of UGCG and MDR1 resulting in increased UGCG protein concentration and P-gp concentration. P-gp functions as an efflux transporter for the cytotoxic agents and works also as a GlcCer flippase in the Golgi apparatus supporting ceramide and GlcCer clearance. The increased GSL production leads to alteration of raft structures in the plasma membrane resulting in increased P-gp and kinase activity. The kinases induce signaling pathways, which lead to increased MDR1 gene transcription. Besides, the altered membrane composition blocks the diffusion of extracellular cytotoxic agents. MDR1 and UGCG are able to influence each other on transcriptional level. The influence of dimerization partners of UGCG in the context of multidrug resistance development is unknown. UGCG = UDP-glucose ceramide glycosyltransferase, MDR1 = multidrug resistance protein 1 , P-gp = P-glycoprotein, GSL = Glycosphingolipid, Gb3 = Globotriasoylceramide

treatment a potential dimer partner of UGCG is not expressed anymore. However, curcumin does also induce necroptosis and is thereby able to revert multidrug resistance [41]. A possible partner of UGCG protein interaction could be the cellular tumor antigen p53. Inhibition of the UGCG by thre-1-Phenyl-2-decanoylamino-3-morpholino-1-propanol hydrochloride (PDMP) or siRNA treatment in p53 deficient human osteosarcoma cells (U20S) leads to increased sensitivity to mitomycin c resulting in ceramide accumulation, decreased cell viability and apoptosis induction shown by increased caspase 3 and 7 activity [42]. p53 deficiency in U2OS cells was accomplished by stable transfection with the E6 oncoprotein of Human Papillomavirus 16 (HPV16), which mediates accelerated degradation of $\mathrm{p} 53$. The data of Haynes et al. may indicate a p53-dependent UGCG regulation (Fig. 2), but studies with p53 proficient cells should be performed in the future. Another interesting target protein for UGCG interaction would be the P-gp protein itself. Furthermore, it has to be clarified in what kind of way the UGCG-derived GSL are involved in apoptotic processes since ceramides induce apoptosis via membrane bound receptors.

\section{Glycosphingolipids on MDR1}

A few studies describe the direct influence of UGCG activity on MDR1 expression, indicating that also gangliosides resulting from UGCG activity may have an effect on MDR1. These studies are described in this chapter. It could be shown that inhibition of the transcription factor nuclear factor (NF) KB results in decreased MDR1 gene and P-gp protein expression [43]. Thereby, NFKB seems to bind directly to the MDR1 promoter through an intronic site (Fig. 3b). In this respect, it is interesting that a ganglioside-dependent activation of NFKB via PKC and NDAPH oxidase could be shown in microglia 
[44] (Fig. 3b). The precursor of all gangliosides is GlcCer generated by the UGCG and accordingly an increased UGCG activity leads to increased gangliosides and/or cerebrosides levels [45] (Figs. 1 and 4). The influence of NFKB on multidrug resistance should be also investigated in other cell types to determine whether or not this is a unique mechanism. Liu et al. showed for the first time that globo series GSL produced by UGCG activity mediate MDR1 expression through $\mathrm{cSrc}$ and $\beta$ catenin signaling [46]. Figure $3 \mathrm{~b}$ shows that UGCG overexpression combined with chemotherapeutic agent treatment leads to increased concentrations of e. g. Gb3 and Gb5 in GSL-enriched microdomains (GEM) resulting in tyrosine kinase cSrc activation and decreased $\beta$ catenin phosphorylation and increased nuclear $\beta$-catenin (Fig. 4). cSrc-induced phosphorylation of GSK-3 $\beta$, which is inactive when phosphorylated and therefore leads no more to phosphorylation of $\beta$-catenin, results in nuclear $\beta$-catenin. The nuclear $\beta$-catenin may bind in a complex with the T-cell factor 4 (Tcf4) to the Tcf4/lymphoid enhancer factor (LEF) binding motif at the MDR1 promoter and thus enhancing promoter activity leading to increased efflux of anticancer drugs of the cell [46]. Another possibility to increase GSK-3 $\beta$ phosphorylation and subsequently reduce GSK- $3 \beta$ activity is to inhibit protein phosphatase 2 (PP2A) activity, which dephosphorylates the GSK-3 $\beta$ protein leading to activation of the protein and degradation of $\beta$-catenin. However, currently, a correlation of $\beta$-arrestin 2 and MDR1 expression could be shown in breast cancer [47]. The protein $\beta$-arrestin 2 is highly expressed in multidrug resistant cells and a knockout of $\beta$-arrestin 2 decreased $M D R 1$ gene expression and P-gp protein expression dramatically leading to increased doxorubicin sensitvity. Since $\beta$-arrestin 2 is able to stabilize $\beta$-catenin by interacting with the intracellular proteins Dishevelled and Axin, an increased expression leads to translocation of $\beta$-catenin to the nucleus, where the previous described mechanisms occur (Fig. 3b). In the future it has also to be investigated whether or not $\beta$-arrestin 2 is able to bind directly to $\beta$-catenin and thereby execute the multidrug resistance inducing effect.

\section{P-gp as a flippase}

The UGCG protein modifies ceramide on the cytosolic surface of the Golgi apparatus (Fig. 4). The conversion of GlcCer to LacCer by adding a galactose molecule is accomplished in the luminal leaflet of the Golgi apparatus. For this reaction GlcCer has to be flipped from the cytosol to the Golgi apparatus lumen. Morad and Cabot postulate that P-gp contributes to multidrug resistance by adopting GlcCer transfer from the Golgi apparatus cytosol to the Golgi lumen in form of a GlcCer flippase and thereby promoting ceramide clearance (reviewed in [48]. Subsequently, ceramides are not able to induce apoptotic signaling pathways anymore. However, De Rosa et al. showed that P-gp activity as a flippase is required for neutral GSL production, but not for acidic GSL (e. g. gangliosides) generation [49]. The hypothesis of the flippase task of P-gp is also supported by the fact that beside ceramides, GlcCer seem to induce toxic effects in the cell [50]. Cer are anabolized to GlcCer, which undergo clearance by flipping and usage for non-toxic complex GSL synthesis (Fig. 4). It is an ongoing discussion whether or not the Cer and GlcCer clearance or the production of complex GSL are the mechanisms to overcome drug sensitivity. The increased GSL production and subsequent transport to cell membranes alters P-gp activity. There are a variety of parameters, which influence the P-gp activity (reviewed in [20]). E. g. the presence of cholesterol, which alters membrane packing and fluidity affects catalytic activity of P-gp and its stimulation by substrates and modulators (Fig. 4). It is to add, that possibly the UGCG structure may allow the GlcCer to be released to the inner as well as to the outer leaflet of the Golgi apparatus. The structure of the ceramide determined by its chain length could determine a preference for the inner or outer leaflet of the cell organell. In this case the cell could discriminate between GlcCer intended for complex GSL synthesis and GlcCer taking the sphingolipid transport protein FAPP2 route to the trans-Golgi apparatus and a flippase would be unnecessary.

\section{Impact of UGCG activity induced alterations of membrane composition by GSL}

Beside the UGCG-P-gp connection, it could be shown that the composition of the cell membrane plays an important role in the induction of multidrug resistance. The question is how UGCG activity can influence P-gp activity not directly, but via GSL synthesis, which lead to alteration of the membrane composition. Tyler et al. showed that the globo-series GSL Gb3 concentration is increased in non-small cell lung cancer (NSCLC) and malignant pleural mesothelioma (MPM) cells, which are cisplatin resistant [51] (Fig. 4). Treatment with the UGCG inhibitor 1-phenyl-2-palmitoylamino-3-morpholino-1-propanol (PPMP) decreased the induced cell surface Gb3, but not P-gp protein expression (MDR1 gene expression pattern similar). Nevertheless, Gb3 silencing restores cisplatin sensitivity, indicating that the UGCG protein does not have a direct effect on P-gp. This leads to the assumption that Gb3 alters membrane composition leading to a changed P-gp activity status. Another study already showed that membrane lipids of drug resistant MCF-7 cells exhibit an increased lipid packing and membrane fluidity as compared to membrane lipids of sensitive cells. Following decitabine treatment these 
biophysical changes in membrane lipids are altered meaning that the packing density is decreased and the membrane fluidity is increased even more [28]. The authors hypothesized that the resistant cells exhibit increased cholesterol-sphingomyelin raft structures, which may influence drug transport and therefore induce multidrug resistance. Ghetie et al. could already show that P-gp is more active in cholesterol and sphingomyelin rich raft structures as compared to a localization outside these membrane structures [52] (Fig. 4). The changes regarding the biophysical properties in the study of Raghavan et al. are blocked by decitabine treatment [28] indicating an epigenetic mechanism meaning methylation-dependent regulation of enzymes of the lipid metabolism in the cell as it has already been shown for the UGCG. Since decitabine inhibits the methylation of all genes in the cell, no detailed information about the molecular mechanisms are available. Peetla et al. reviewed in 2013 that in drug resistant cell membranes the sphingolipid and/or cholesterol content is increased leading to a higher membrane order and less permeability resulting in reduced lipophilic drug diffusion and less effective cytotoxic substances [53] independent from P-gp (Fig. 4). This shows, not only the impact on membrane proteins is altered by changed lipid composition, but also the behavior of the toxic substance in the lipid bilayer.

\section{Conclusions}

The influence of the UGCG on multidrug resistance was investigated in depth for the last two decades. But still many pieces of the puzzle need to be analyzed. The link between UGCG overexpression and multidrug resistance induction is more complex than it was anticipated. Particularly, the influence of membrane properties should be investigated in future studies since during malignant transformation of cells the biophysical parameters of the membranes changes.

\footnotetext{
Abbreviations

ABCB1: ATP-binding cassette sub-family B member 1; Bcl-2: B-cell lymphoma 2; BCl-xL: B-cell lymphoma-extra large; Cer: Ceramide; CerS6: Ceramide synthase 6; ChIP: Chromatin immunoprecipitation; CRISPR: Clustered Regularly Interspaced Short Palindromic Repeats; CS: CRISPR score; cSrc: Tyrosine kinase; DNMT3a: DNA (cytosine-5)-methyltransferase 3a; E2F1: E2F transcription factor 1; EMSA: Electrophoretic mobility shift assay; ER: Estrogen receptor; ERK: Extracellular-signal regulated kinase; FAN: Factor associated with activation of neutral sphingomyelinase; FBS: Fetal bovine serum; Gb3: Globotriaosylceramide; GEM: Glycosphingolipid-enriched microdomain; GLA: a-galactosidase A gene; GlcCer: Glycosylceramide; GSK3: Glycogen synthase kinase 3; GSL: Glycosphingolipid; LacCer: Lactosylceramide; LEF: Tcf4/lymphoid enhancer factor; MDR1: Multidrug resistance protein 1 gene; NFkB: Nuclear factor (NF) kB; NSD: Neutral SMase domain; PDMP: Thre-1-Phenyl-2-decanoyl-amino-3morpholino-1-propanol hydrochloride; P-gp: P-glycoprotein; PPMP: 1-phenyl2-palmitoylamino-3-morpholino-1-propanol; ROS: Reactive oxygen specie; SMase: Sphingomyelinase; Tcf4: T-cell factor 4; TNFR1: Tumor necrosis factor receptor 1; TNFa: Tumor necrosis factor a; UBE2R1: Ubiquitin-activating enzyme E2 R1; UGCG: UDP-Glucose ceramide glycosyltransferase: Wnt: Wingless
}

\section{Acknowledgements}

The knockdown of the UGCG by CRISPR-Cas was kindly performed by Martin Wegner from the working group Manuel Kaulich (Institute for Biochemistry II, University Clinic Frankfurt am Main). Matti Kjellberg is acknowledged for the HPTLC work

\section{Funding}

This work was funded by the Deutsche Forschungsgemeinschaft (DFG) (WE 5825/1-1; MSW) and the SFB 1039, the August Scheidel-Stiftung and the Heinrich und Fritz Riese-Stiftung and the Sigrid Jusélius Foundation.

\section{Availability of data and materials}

The publications quoted are publically available from the publishers. All data supporting the fact that the UGCG is a unique protein in cells are presented within the manuscript. Raw data as required may be requested from the corresponding author.

\section{Authors' contributions}

All authors have read and approved the final version of this manuscript. MSW wrote the main parts of all sections. LG added the section Materials and Methods and illustrated Figs. 2 and 3. PM analyzed and interpreted the HPTLC data and added valuable information regarding the flippase function. SG and GG edited the manuscript.

Ethics approval and consent to participate Not applicable.

\section{Consent for publication}

Not applicable.

\section{Competing interests}

The authors declare that they have no competing interests.

\section{Publisher's Note}

Springer Nature remains neutral with regard to jurisdictional claims in published maps and institutional affiliations.

\section{Author details}

1 pharmazentrum frankfurt/ ZAFES, Institute of Clinical Pharmacology, Johann Wolfgang Goethe-University, House 74, Theodor Stern-Kai 7, 60590 Frankfurt am Main, Germany. ${ }^{2}$ Biochemistry, Faculty of Science and Engineering, Åbo Akademi University, Artillerigatan 6A, III, BioCity, Fl-20520 Turku, Finland.

Received: 2 August 2017 Accepted: 31 January 2018

Published online: 06 February 2018

\section{References}

1. Ichikawa S, Sakiyama H, Suzuki G, Hidari Kl, Hirabayashi Y. Expression cloning of a cDNA for human ceramide glucosyltransferase that catalyzes the first glycosylation step of glycosphingolipid synthesis. Proc Natl Acad Sci U S A. 1996:93:12654.

2. D'Angelo G, Capasso S, Sticco L, Russo D. Glycosphingolipids: synthesis and functions. FEBS J. 2013;280:6338-53.

3. Sandhoff K, Kolter T. Biochemistry of glycosphingolipid degradation. Clin Chim Acta. 1997;266:51-61

4. Yamashita T, Wada R, Sasaki T, Deng C, Bierfreund U, Sandhoff K, Proia RL. A vital role for glycosphingolipid synthesis during development and differentiation. Proc Natl Acad Sci U S A. 1999:96:9142-7.

5. Stirnemann J, Belmatoug N, Camou F, Serratrice C, Froissart R, Caillaud C, Levade T, Astudillo L, Serratrice J, Brassier A, Rose C, Billette de Villemeur T, Berger MG. A review of Gaucher disease pathophysiology, clinical presentation and treatments. Int J Mol Sci. 2017:18:E441.

6. Schiffmann R, Fuller M, Clarke LA, Aerts JM. Is it Fabry disease? Genet Med. 2016;18:1181-5.

7. Sandhoff K. Neuronal sphingolipidoses: membrane lipids and sphingolipid activator proteins regulate lysosomal sphingolipid catabolism. Biochimie. 2016:130:146-51.

8. Hashimoto N, Matsumoto I, Takahashi H, Ashikawa H, Nakamura H, Murayama T. Cholesterol-dependent increases in glucosylceramide synthase activity in Niemann-pick disease type C model cells: abnormal trafficking of 
endogenously formed ceramide metabolites by inhibition of the enzyme. Neuropharmacology. 2016;110:458-69.

9. Ishibashi Y, Kohyama-Koganeya A, Hirabayashi Y. New insights on glucosylated lipids: metabolism and functions. Biochim Biophys Acta. 2013;1831:1475-85.

10. Jennemann R, Grone HJ. Cell-specific in vivo functions of glycosphingolipids: lessons from genetic deletions of enzymes involved in glycosphingolipid synthesis. Prog Lipid Res. 2013;52:231-48.

11. Liu YY, Hill RA, Li YT. Ceramide glycosylation catalyzed by glucosylceramide synthase and cancer drug resistance. Adv Cancer Res. 2013;117:59-89.

12. Sun Y, Zhang T, Gao P, Meng B, Gao Y, Wang X, Zhang J, Wang H, Wu X, Zheng W, Zhou G. Targeting glucosylceramide synthase downregulates expression of the multidrug resistance gene MDR1 and sensitizes breast carcinoma cells to anticancer drugs. Breast Cancer Res Treat. 2010;121:591-9.

13. Wang T, Birsoy K, Hughes NW, Krupczak KM, Post Y, Wei JJ, Lander ES, Sabatini DM. Identification and characterization of essential genes in the human genome. Science. 2015;350:1096-101.

14. Jennemann R, Sandhoff R, Langbein L, Kaden S, Rothermel U, Gallala H, Sandhoff K, Wiegandt H, Grone HJ. Integrity and barrier function of the epidermis critically depend on glucosylceramide synthesis. J Biol Chem. 2007;282:3083-94

15. Amen N, Mathow D, Rabionet M, Sandhoff R, Langbein L, Gretz N, Jackel C, Grone HJ, Jennemann R. Differentiation of epidermal keratinocytes is dependent on glucosylceramide:ceramide processing. Hum Mol Genet. 2013;22:4164-79.

16. Liu YY, Patwardhan GA, Xie P, Gu X, Giuliano AE, Cabot MC. Glucosylceramide synthase, a factor in modulating drug resistance, is overexpressed in metastatic breast carcinoma. Int J Oncol. 2011;39:425-31.

17. Gouaze V, Yu JY, Bleicher RJ, Han TY, Liu YY, Wang H, Gottesman MM, Bitterman A, Giuliano AE, Cabot MC. Overexpression of glucosylceramide synthase and P-glycoprotein in cancer cells selected for resistance to natural product chemotherapy. Mol Cancer Ther. 2004;3:633-9.

18. Gouaze-Andersson V, Yu JY, Kreitenberg AJ, Bielawska A, Giuliano AE, Cabot MC. Ceramide and glucosylceramide upregulate expression of the multidrug resistance gene MDR1 in cancer cells. Biochim Biophys Acta. 2007;1771:1407-17.

19. Katayama K, Noguchi K, Sugimoto Y. Regulations of P-glycoprotein/ABCB1/ MDR1 in human cancer cells. New Journal of Science. 2014;2014:10.

20. Sharom FJ. Complex interplay between the P-glycoprotein multidrug efflux pump and the membrane: its role in modulating protein function. Front Oncol. 2014:4:41.

21. Efferth T, Volm M. Multiple resistance to carcinogens and xenobiotics: Pglycoproteins as universal detoxifiers. Arch Toxicol. 2017;91(7):2515-38.

22. Chen $\mathrm{KG}$, Sikic BI. Molecular pathways: regulation and therapeutic implications of multidrug resistance. Clin Cancer Res. 2012;18:1863-9.

23. Huang WC, Chen CL, Lin YS, Lin CF. Apoptotic sphingolipid ceramide in cancer therapy. J Lipids. 2011;2011:565316.

24. Hernandez-Corbacho MJ, Salama MF, Canals D, Senkal CE, Obeid LM. Sphingolipids in mitochondria. Biochim Biophys Acta. 2017;1862:56-68.

25. Liu J, Zhang X, Liu A, Zhang D, Su Y, Liu Y, You D, Yuan L, Kong X, Wang X, Sun $P$. Altered methylation of glucosylceramide synthase promoter regulates its expression and associates with acquired multidrug resistance in invasive ductal breast cancer. Oncotarget. 2016;7:36755-66.

26. Liu J, Sun P, Sun Y, Liu A, You D, Jiang F. Expression of glucosylceramide synthase in invasive ductal breast cancer may be correlated with high estrogen receptor status and low HER-2 status. Diagn Pathol. 2014:9:22.

27. Kandoth C, McLellan MD, Vandin F, Ye K, Niu B, Lu C, Xie M, Zhang Q, McMichael JF, Wyczalkowski MA, Leiserson MD, Miller CA, Welch JS, Walter MJ, Wendl MC, Ley TJ, Wilson RK, Raphael BJ, Ding L. Mutational landscape and significance across 12 major cancer types. Nature. 2013;502:333-9.

28. Raghavan V, Vijayaraghavalu S, Peetla C, Yamada M, Morisada M, Labhasetwar V. Sustained epigenetic drug delivery depletes cholesterolsphingomyelin rafts from resistant breast cancer cells, influencing biophysical characteristics of membrane lipids. Langmuir. 2015;31:11564-73.

29. Zhang X, Wu X, Su P, Gao Y, Meng B, Sun Y, Li L, Zhou Z, Zhou G. Doxorubicin influences the expression of glucosylceramide synthase in invasive ductal breast cancer. PLoS One. 2012;7:e48492

30. Ikeda K, Horie-Inoue K, Inoue S. Identification of estrogen-responsive genes based on the DNA binding properties of estrogen receptors using highthroughput sequencing technology. Acta Pharmacol Sin. 2015;36:24-31.

31. Cui J, Shen Y, Li R. Estrogen synthesis and signaling pathways during aging: from periphery to brain. Trends Mol Med. 2013;19:197-209.
32. Zhang X, Wu X, Li J, Sun Y, Gao P, Zhang C, Zhang H, Zhou G. MDR1 (multidrug resistence 1) can regulate GCS (glucosylceramide synthase) in breast cancer cells. J Surg Oncol. 2011;104:466-71.

33. Wang Q, Zou J, Zhang X, Mu H, Yin Y, Xie P. Glucosylceramide synthase promotes $\mathrm{BCl}-2$ expression via the ERK signaling pathway in the K562/A02 leukemia drug-resistant cell line. Int J Hematol. 2014;100:559-66.

34. Chiu WH, Su WC, Li CL, Chen CL, Lin CF. An increase in glucosylceramide synthase induces $\mathrm{BCl}-\mathrm{xL}$-mediated cell survival in vinorelbine-resistant lung adenocarcinoma cells. Oncotarget. 2015;6:20513-24.

35. Liu YY, Han TY, Giuliano AE, Cabot MC. Expression of glucosylceramide synthase, converting ceramide to glucosylceramide, confers adriamycin resistance in human breast cancer cells. J Biol Chem. 1999;274:1140-6.

36. Marks DL, Wu K, Paul P, Kamisaka Y, Watanabe R, Pagano RE. Oligomerization and topology of the Golgi membrane protein glucosylceramide synthase. J Biol Chem. 1999:274:451-6.

37. Crespo PM, Silvestre DC, Gil GA, Maccioni HJ, Daniotti UL, Caputto BL. C-Fos activates glucosylceramide synthase and glycolipid synthesis in PC12 cells. J Biol Chem. 2008;283:31163-71.

38. Di Sano F, Fazi B, Citro G, Lovat PE, Cesareni G, Piacentini M. Glucosylceramide synthase and its functional interaction with RTN-1C regulate chemotherapeutic-induced apoptosis in neuroepithelioma cells. Cancer Res. 2003;63:3860-5.

39. Shakor AB, Atia M, Ismail IA, Alshehri A, El-Refaey H, Kwiatkowska K, Sobota A. Curcumin induces apoptosis of multidrug-resistant human leukemia HL60 cells by complex pathways leading to ceramide accumulation. Biochim Biophys Acta. 2014;1841:1672-82.

40. Chen H, Duan J, Zuo F. Mechanism of the reversal effect of mifepristone on drug resistance of the human cervical cancer cell line HeLa/MMC. Genet Mol Res. 2014;13:1288-95.

41. Li F, Chen X, Xu B, Zhou H. Curcumin induces p53-independent necrosis in H1299 cells via a mitochondria-associated pathway. Mol Med Rep. 2015;12: 7806-14.

42. Haynes TA, Filippov V, Filippova M, Yang J, Zhang K, Duerksen-Hughes PJ. DNA damage induces down-regulation of UDP-glucose ceramide glucosyltransferase, increases ceramide levels and triggers apoptosis in p53deficient cancer cells. Biochim Biophys Acta. 2012;1821:943-53.

43. Bentires-Alj M, Barbu V, Fillet M, Chariot A, Relic B, Jacobs N, Gielen J, Merville MP, Bours V. NF-kappaB transcription factor induces drug resistance through MDR1 expression in cancer cells. Oncogene. 2003;22:90-7.

44. Min KJ, Pyo HK, Yang MS, Ji KA, Jou I, Joe EH. Gangliosides activate microglia via protein kinase C and NADPH oxidase. Glia. 2004;48:197-206.

45. Gouaze V, Liu YY, Prickett CS, Yu JY, Giuliano AE, Cabot MC Glucosylceramide synthase blockade down-regulates P-glycoprotein and resensitizes multidrug-resistant breast cancer cells to anticancer drugs. Cancer Res. 2005;65:3861-7.

46. Liu YY, Gupta V, Patwardhan GA, Bhinge K, Zhao Y, Bao J, Mehendale H, Cabot MC, Li YT, Jazwinski SM. Glucosylceramide synthase upregulates MDR1 expression in the regulation of cancer drug resistance through cSrc and beta-catenin signaling. Mol Cancer. 2010;9:145.

47. Jing $X$, Zhang H, Hu J, Su P, Zhang W, Jia M, Cheng H, Li W, Zhou G. Betaarrestin 2 is associated with multidrug resistance in breast cancer cells through regulating MDR1 gene expression. Int J Clin Exp Pathol. 2015;8:1354-63.

48. Morad SA, Cabot MC. Tamoxifen regulation of sphingolipid metabolismtherapeutic implications. Biochim Biophys Acta. 2015;1851:1134-45.

49. De Rosa MF, Sillence D, Ackerley C, Lingwood C. Role of multiple drug resistance protein 1 in neutral but not acidic glycosphingolipid biosynthesis. J Biol Chem. 2004;279:7867-76

50. Dahdouh F, Raane M, Thevenod F, Lee WK. Nickel-induced cell death and survival pathways in cultured renal proximal tubule cells: roles of reactive oxygen species, ceramide and ABCB1. Arch Toxicol. 2014;88:881-92.

51. Tyler A, Johansson A, Karlsson T, Gudey SK, Brannstrom T, Grankvist K, BehnamMotlagh P. Targeting glucosylceramide synthase induction of cell surface globotriaosylceramide (Gb3) in acquired cisplatin-resistance of lung cancer and malignant pleural mesothelioma cells. Exp Cell Res. 2015;336:23-32.

52. Ghetie MA, Marches R, Kufert S, Vitetta ES. An anti-CD19 antibody inhibits the interaction between P-glycoprotein (P-gp) and CD19, causes P-gp to translocate out of lipid rafts, and chemosensitizes a multidrug-resistant (MDR) lymphoma cell line. Blood. 2004;104:178-83.

53. Peetla C, Vijayaraghavalu S, Labhasetwar V. Biophysics of cell membrane lipids in cancer drug resistance: implications for drug transport and drug delivery with nanoparticles. Adv Drug Deliv Rev. 2013;65:1686-98. 